\title{
Tecnología para la obtención de polvos microporosos de acero inoxidable $e^{(\cdot)}$
}

\author{
M. Martínez $z^{*}$ W. Suwardjo*, B. Aragón ${ }^{* *}$, L. García ${ }^{* *}$, A. Formoso ${ }^{* * *}$ y A. Cortée ${ }^{* * *}$
}

Resumen En el trabajo se trata el desarrollo de la tecnología de obtención de polvos microporosos de acero inoxidable mediante atomización y recocido de descarburación. La esencia del proceso consiste en recarburar el metal en estado líquido y, posteriormente, descarburar el polvo en estado sólido mediante recocido en hidrógeno o amoniaco disociado. Con esta tecnología se logra la formación de una microporosidad interna en la partícula, que aligera el material y mejora los procesos de conformación, ya que aumenta la deformación que puede experimentar una partícula. A su vez, se reduce el costo de producción y los gastos de inversión, para asimilar la tecnología. Además, se exponen los resultados del estudio cinético acerca de la descarburación y la caracterización del polvo de acero inoxidable obtenido.

Palabras clave Pulvimetalurgia. Atomización. Polvo de acero inoxidable. Microporosidad. Recocido de descarburación.

\section{A technology for obtaining microporous stainless steel powder}

\begin{abstract}
In this paper, a technology for obtaining microporous stainless steel powders by atomization and decarburization annealing is dealt with. The essence of the process is based in the recarburisation of the liquid-state metal and a subsequent decarburization of the solid-state powder by means of annealing in hydrogen or dissociated ammonia. An internal microporosity in the particle is formed through this technology activating the pressing process due to the improvement of its plastic behaviour, while a reduction in both production costs and investment expenses for introducing this technology takes place. In addition, the results of the study regarding decarburization, as well as the characterization of stainless steel powders are shown.
\end{abstract}

Keywords Powder metallurgy. Atomization. Stainless steel powders. Microporosity. Decarburization annealing.

\section{INTRODUCCIÓN}

La necesidad del Taller Experimental-Productivo de Pulvimetalurgia (TEPP), subordinado al Centro de Investigaciones Metalúrgicas (CIME), de dar respuesta a las demandas del mercado interno y externo de polvos de acero inoxidable, estimuló la búsqueda de una tecnología que empleara las materias primas nacionales para su fabricación, de forma tal, que fuera de factible asimilación y, a su vez, que el polvo tuviese una calidad comparable a la de los disponibles en el mercado internacional.
Se debe destacar que Cuba dispone de materias primas para la fabricación de acero inoxidable, entre las que se pueden mencionar: sínter de níquel, semiproductos de acero inoxidable de la factoría ACINOX Tunas, chatarra de acero al carbono e inoxidable y ferrocromo. Por otra parte, el TEPP cuenta con instalaciones para la fabricación de polvos de aleaciones base cobre, níquel y hierro.

Debido a esto, se planteó la posibilidad de fabricación de polvo de acero inoxidable recarburando la aleación madre en estado líquido hasta la concentración eutéctica $(4,2-5,0 \%$ en masa de C) en

(•) Trabajo recibido el día 19 de enero de 2001 y aceptado en su forma final el día 23 de julio de 2001.

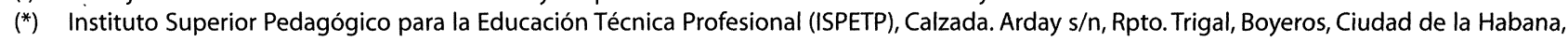
Cuba.

(**) Centro de Investigaciones Metalúrgicas (CIME), Avda. 51, 23611, La Lisa, Ciudad de la Habana, Cuba.

${ }^{* * *}$ ) Centro Nacional de Investigaciones Metalúrgicas (CENIM), Avda. Gregorio del Amo, 8. 28040 Madrid (España). 
un horno de inducción y, después de la atomización, descarburando el polvo en estado sólido mediante un recocido en una atmósfera de hidrógeno o amoniaco disociado mediante las reacciones:

$$
\begin{array}{ll}
\mathrm{C}+2 \mathrm{H}_{2} \rightarrow \mathrm{CH}_{4} & \Delta \mathrm{H}=-74,848 \mathrm{~kJ} / \mathrm{mol} \\
3 \mathrm{C}+4 \mathrm{NH}_{3} \rightarrow 3 \mathrm{CH}_{4}+2 \mathrm{~N}_{2} & \Delta \mathrm{H}=-40,857 \mathrm{~kJ} / \mathrm{mol}
\end{array}
$$

La difusión del carbono debe originar una estructura microporosa que disminuye la masa de la partícula y mejora su compresibilidad. Además, el polvo en estado de atomización con alto carbono y elevada tensión estructural podría ser fácilmente triturado, al ser muy frágil, con lo que se elevaría el rendimiento de la fracción útil $(<180 \mu \mathrm{m}$ u 80 mesh) a prácticamente un $100 \%$.

Esta idea surge del análisis de un trabajo presentado, en 1977, por Shorniak y Olikier ${ }^{[1]}$, en el que exponen los resultados acerca del recocido en hidrógeno de polvos de hierro. En este trabajo se analizan distintos factores que influyen en el proceso de descarburación, tales como tiempo de retención y temperatura de calentamiento, concluyendo que el contenido de carbono puede descender desde un 3,39 a un 0,006 \% en masa.

Posteriormente, en 1985, Rabinovich y Zaslovskii ${ }^{[2]}$ publican los resultados sobre el incremento de la resistencia físico-mecánica de los aceros inoxidables austeníticos mediante la descarburación en fase sólida y nitruración. En este caso, los ensayos se realizaron sobre productos laminados tubulares, para los que el contenido en carbono disminuyó del 0,026-0,048 \% al 0,015 \%. Para ello, los autores combinan un tratamiento térmico en alto vacío con adición de cascarilla de laminación, para estimular el potencial descarburante. Estos resultados muestran que los aceros de alto contenido de cromo pueden ser descarburados en condiciones económicamente permisibles.

Los primeros trabajos sobre el aumento del contenido de carbono en el polvo de acero inoxidable para mejorar sus propiedades datan de la década de los años 50.

La mayoría de los autores han optado por introducir el carbono en el polvo de acero inoxidable mediante un proceso de mezcla. En este sentido, en 1956, se registra una patente inglesa ${ }^{[3]}$ en la que se mezcla polvo de acero inoxidable con 0,5-1,25 $\% \mathrm{C}$, con el fin de ampliar suficientemente el rango de temperaturas de sólido-líquido y facilitar el control de temperaturas durante la sinterización. Posteriormente, el carbono es eliminado mediante un tratamiento de descarburación. Esta técnica so- lo es aplicable a la fabricación de filtros que, debido a su porosidad, permiten el paso del agente descarburante. En el mismo año, se publica también una patente alemana ${ }^{[4]}$ que señala la introducción del carbono mediante un polvo finamente triturado de aleación eutéctica o polvos de diversos elementos en proporciones que den lugar a un eutéctico, con el fin de disminuir la temperatura de sinterización. En 1990, unas patentes japonesas $^{[5}$ y 6$]$ ofrecen distintas expresiones matemáticas que relacionan los contenidos de carbono y oxígeno que garantizan la desoxidación durante la sinterización.

En 1991, en otra patente japonesa ${ }^{[7]}$ se plantea que esta relación $\mathrm{C} / \mathrm{O}$ debe ser como mínimo equivalente o mayor a su relación atómica. Sin embargo, una alternativa a esta relación se ofreció por los investigadores daneses Larsen y Thorsen en un trabajo presentado en el Congreso Mundial de Pulvimetalurgia de $1993^{[8]}$, donde, con el fin de elevar el poder reductor del hidrógeno durante la sinterización, proponen mezclar el polvo de acero inoxidable con $0,1 \%$ de polvo de grafito.

En 1993, se publica otra patente japonesa ${ }^{[9]}$ que, propone enriquecer la atmósfera de hidrógeno que se utiliza durante la sinterización con un 0,01$5,0 \%$ de un hidrocarburo gaseoso (metano, acetileno u otro). De esta forma, se disminuye la oxidación superficial del polvo y se mejora la sinterización, debido a la deposición de carbono atómico en la superficie del polvo. Sin embargo, las condiciones de la atomización del polvo y su prensado siguen siendo limitadas en cuanto a temperaturas y presiones necesarias, respectivamente.

En 1996, J. Saunders publica una patente ${ }^{[10]}$ en la que propone, para elevar los niveles de resistencia al desgaste y a la corrosión, la adición de polvo de grafito libre a los polvos de acero inoxidable mediante mezclado. Sin embargo, todas estas adiciones mecánicas de carbono, en una u otra proporción, no permiten elevar la microporosidad interna de la partícula, además de que no ejercen ninguna influencia en las condiciones de la atomización.

El único trabajo encontrado, donde el carbono se introduce en la fusión, antes de la atomización, es la patente presentada en 1958 por Probst y Le Brasse $^{[11]}$. Sin embargo, la adición propuesta de 0,5-1,25\% de carbono es baja para hacer disminuir apreciablemente la temperatura de fusión y no facilita la molienda del polvo, puesto que con estos contenidos de carbono el polvo es aún resistente a la fracturación. Como el objetivo 
propuesto por los autores es efectuar simultáneamente los procesos de sinterización y descarburación (completa o casi completa), se ha de garantizar una adecuada interacción de la partícula con el agente descarburante. Por ello, en la práctica, este método se aplica casi exclusivamente a la obtención de filtros, ya que no se forma microporosidad interna en la partícula.

En la literatura especializada, son conocidas las altas exigencias tecnológicas necesarias para la obtención de polvos de acero inoxidable, así como para su prensado y sinterización.

Para la obtención del polvo por atomización se requieren altas temperaturas de colada $\left(>1.700^{\circ} \mathrm{C}\right)$ y altas presiones del fluído que se utiliza. Por ejemplo, según el ASM Committee on Production of Steel Powders ${ }^{[12]}$, cuando se emplea el agua para obtener fracciones menores de $177 \mu \mathrm{m}$ (80 mesh) se emplean presiones de $14 \mathrm{MPa}$.

Según Stevenson ${ }^{[13]}$, para la compactación de los polvos de acero inoxidable a escala industrial se suelen emplear presiones de compactación de 550-830 MPa y temperaturas de sinterización entre $1.120-1.150^{\circ} \mathrm{C}$, aunque en algunos casos pueden superar los $1.315^{\circ} \mathrm{C}$. También se emplean procesos más avanzados y complejos, como la extrusión y sinterización en caliente y el moldeo por inyección, entre otros.

En el presente trabajo se ha desarrollado un proceso para la fabricación de polvos de acero inoxidable en dos etapas. En una primera etapa, se obtuvo polvo de acero con un elevado contenido de carbono por atomización y, en una segunda etapa, se sometió este polvo a un recocido de descarburización en atmósfera de hidrógeno.

\section{PARTE EXPERIMENTAL}

Para la realización de los objetivos propuestos se llevó a cabo un plan experimental para caracterizar la atomización, la descarburación y la eficiencia de la molienda.

\subsection{Atomización}

El polvo de hierro aleado se produce mediante la atomización de la aleación fundida con agua a alta presión o con gas nitrógeno. En la figura 1 se muestra el diagrama del proceso y en la figura 2 el esquema de la instalación de atomización del taller de pulvimetalurgia del CIME, que también se utiliza para la fabricación de polvo de bronce ${ }^{[14]}$.

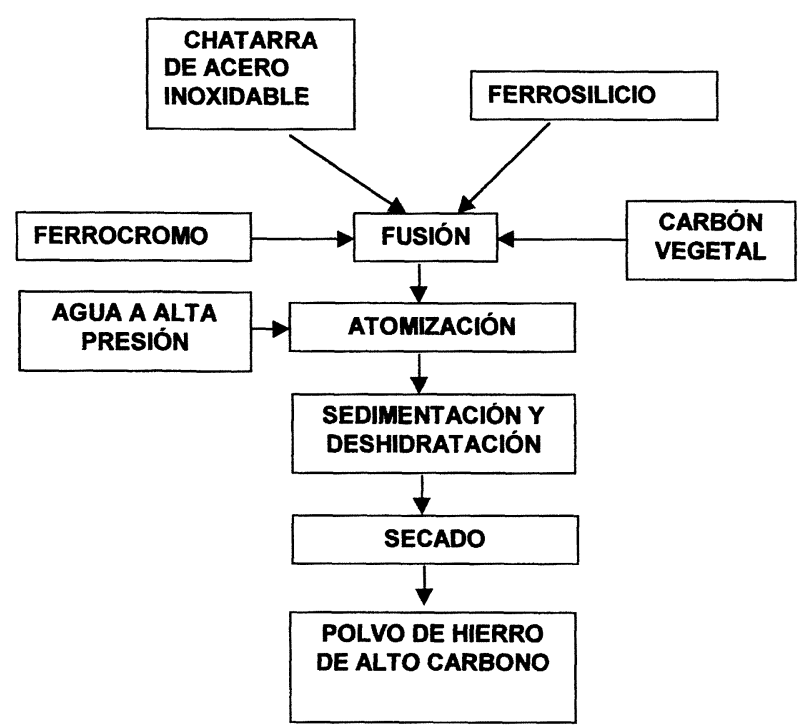

Figura 1. Esquema general del proceso de atomización.

Figure 1. General layout of the atomization process.

Se realizaron tres ensayos de atomización bajo los parámetros que se presentan en la tabla I. Se empleó un horno de inducción para la fusión de la chatarra de acero inoxidable tipo 304, con adición de ferroaleaciones para compensar las pérdidas de $\mathrm{Cr}$ y $\mathrm{Si}$, así como carbón vegetal para saturar de carbono la aleación. La temperatura de colada se mantuvo entre 1.550 y $1.650^{\circ} \mathrm{C}$ y el enfriamiento del polvo se efectuó en agua (cámara húmeda). Teniendo en cuenta las recomendaciones de $\mathrm{Ny}$ borg $^{[15]}$, se varía el contenido de silicio para evaluar el comportamiento de la resistencia a la oxidación durante la atomización.

Al comparar las condiciones en que se realizaron las tres atomizaciones (Tabla I), se observa un incremento paulatino en la formación de la partícula (mayores presiones y menores diámetros del chorro metálico) y, asimismo, del control de la composición química, ya para la tercera atomización no se adicionó ferromanganeso. Los contenidos en fósforo y el azufre, no indicados en la tabla I, se mantuvieron bajos en las tres atomizaciones, o sea,entre 0,03 y $0,007 \%$, en masa.

En la tabla II se ofrecen los resultados de los ensayos de atomización. Para caracterizar los polvos obtenidos mediante análisis granulométrico y determinaciones de fluidez, densidad aparente y compresibilidad, se emplearon las Normas ISO 4497:83, ISO 4490:78, ISO 3923/1:77 e ISO 3927:85, respectivamente.

Como se observa en la tabla II, la variación de los parámetros de las atomizaciones ofrecen una 


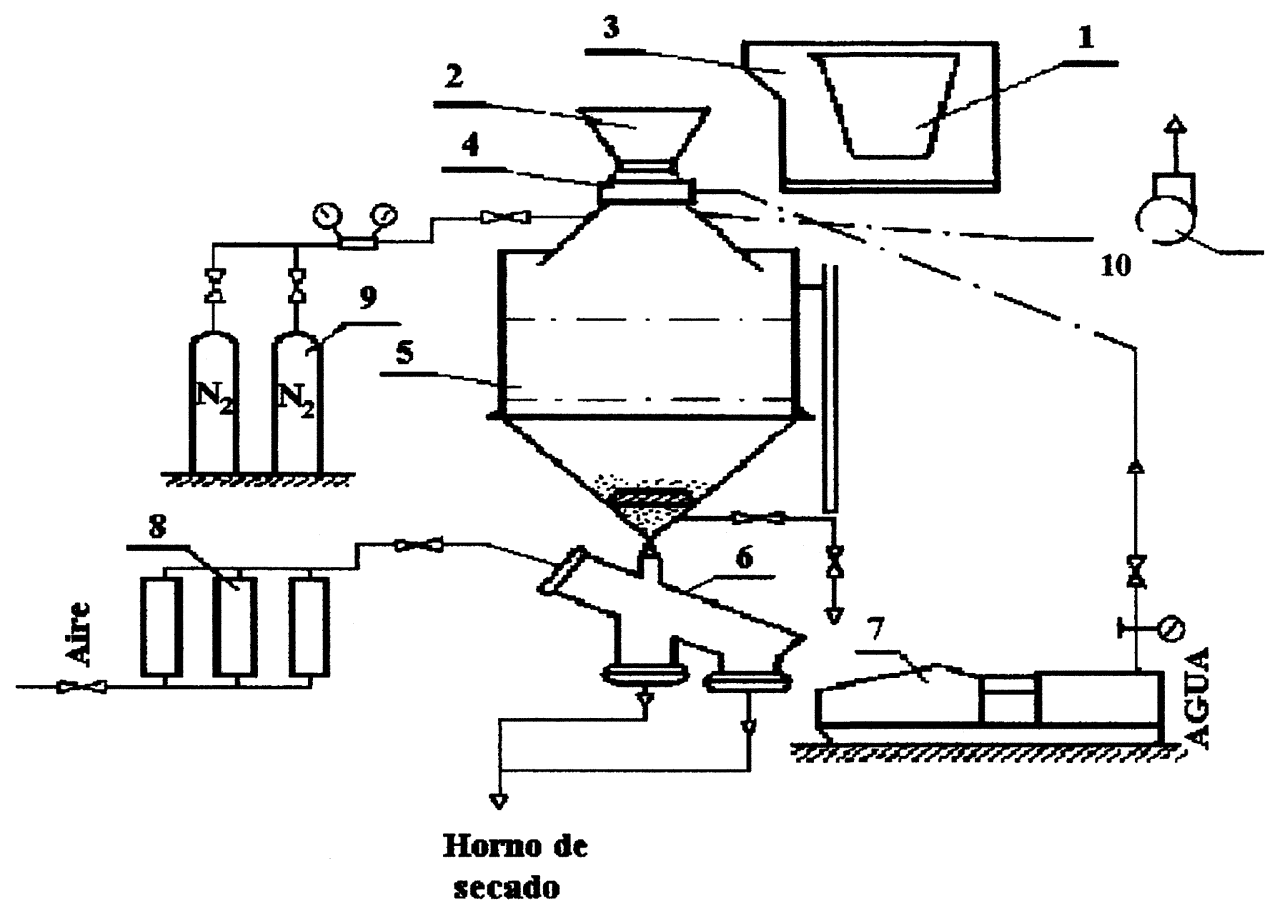

Figura 2. Esquema de la instalación de atomización: 1) Crisol de grafito, 2) Colector de metal líquido, 3) Horno de inducción, 4) Inyector del fluido de atomización, 5) Cámara de atomización, 6) Colector de polvo, 7) Bomba de émbolo, 8) Calentador de aire, 9) Batería botellas de nitrógeno, 10) Extractor.

Figure 2. Scheme of the atomization plant: 1) Graphite melting crucible, 2) Liquid metal receiver, 3) Induction furnace, 4) Spraying injector, 5) Atomization chamber, 6) Powder collector, /9 Piston pump, 8) Blast heater, 9) Battery of nitrogen bottles, 10) Exhausting device.

Tabla I. Parámetros experimentales utilizados para la atomización

Table I. Experimental data used for the atomization

\begin{tabular}{|c|c|c|c|}
\hline \multirow{2}{*}{$\begin{array}{l}\text { Parámetros de } \\
\text { la atomización }\end{array}$} & \multicolumn{3}{|c|}{ Atomización } \\
\hline & $\mathbf{I}$ & II & III \\
\hline Tipo de fluido & $\mathrm{N}_{2}$ & $\mathrm{H}_{2} \mathrm{O}$ & $\mathrm{H}_{2} \mathrm{O}$ \\
\hline Presión del fluido, $\mathrm{MPa}$ & 0,35 & 9 & 9 \\
\hline Diámetro del chorro metálico, $\mathrm{mm}$ & 10 & 12 & 10 \\
\hline Temperatura de colada, ${ }^{\circ} \mathrm{C}$ & 1.600 & $1.600 \quad 1$ & 1.600 \\
\hline \multicolumn{4}{|l|}{ Composición, \%: } \\
\hline C & 4,68 & $>4,6$ & $>4,8$ \\
\hline $\mathrm{Si}$ & 1,5 & 0,13 & 1,43 \\
\hline $\mathrm{Mn}$ & $>1,5$ & 1,04 & 0,87 \\
\hline $\mathrm{Cr}$ & 20,0 & 15,66 & 18,74 \\
\hline $\mathrm{Ni}$ & 10,0 & 11,8 & 8,00 \\
\hline
\end{tabular}

disminución del tamaño medio de la partícula e incrementan el rendimiento de la fracción útil, mientras que la fluidez disminuye y la densidad aparente toma un valor de 2,51-2,58 $\mathrm{g} / \mathrm{cm}^{3}$ para las condiciones más críticas. Por otra parte, el grado de oxidación (evaluado a través del brillo metálico) se mantiene bajo, con contenidos de silicio entre 1,4 y $1,5 \%$, independientemente del fluído empleado para la atomización. En la figura 3 se muestra la morfología de una partícula de polvo de la tercera atomización y, se puede observar, la forma irregular típica de las partículas atomizadas por agua y una superficie lisa y brillante.

\subsection{Descarburación}

Los ensayos de descarburación se llevaron a cabo con muestras de polvo de la tercera atomización, puesto que, por su contenido de silicio alto y manganeso bajo, favorece mayor difusión del carbono que en las otras aleaciones. El silicio aumenta la actividad del carbono por lo que su presencia en este caso será positiva. Por otra parte, como el manganeso es formador de carburos, aunque son de enlace más débil que los carburos de cromo presentes en esta aleación, su presencia influirá 
Tabla II. Resultados de los ensayos de caracterización de los polvos atomizados

Table II. Results of the characterization test of the atomized powders

\begin{tabular}{lccc}
\hline Caracterización del polvo atomizado & I & \multicolumn{2}{c}{ Atomización } \\
II & III \\
\hline Tamaño medio de la fracción útil, $\mu \mathrm{m}$ & 133 & 86 & 83 \\
Rendimiento de la fracción útil, \% & 14 & 40 & 67 \\
Fluidez, $\mathrm{S} / 50 \mathrm{~g}$ & 45 & 48 & 57 \\
Densidad aparente, $\mathrm{g} / \mathrm{cm}^{3}$ & 2,88 & 2,51 & 2,58 \\
Compresibilidad para $900 \mathrm{MPa}, \mathrm{g} / \mathrm{cm}^{3}$ & 5,45 & 5,96 & 5,25 \\
Morfología & Irregular, ligamentos, & Irregular, ligamentos, & Irregular, ligamentos, \\
& liso, brillo metálico & rugoso, abundante orín & liso, brillo metálico \\
& & & \\
\hline
\end{tabular}

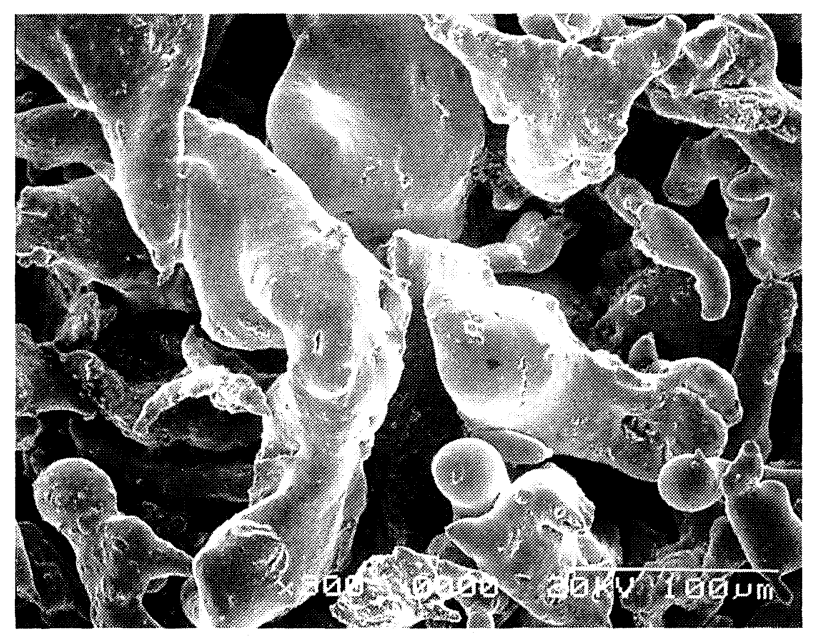

Figura 3. Morfología del polvo atomizado, $\times 300$.

Figure 3. Powder morphology before decarburization, $\times 300$.

negativamente en la migración del carbono hacia la interfase sólido-gas.

En los inicios de la descarburación no deben emplearse temperaturas superiores a $950^{\circ} \mathrm{C}$, puesto que se produce una cierta sinterización que obligará a una posterior molienda. Esta molienda será engorrosa por la mayor ductibilidad que adquiere el polvo al disminuir el contenido de carbono. Sin embargo, esta temperatura es insuficiente para activar el flujo de carbono a niveles suficientemente económicos. Por consiguiente, para resolver ambos problemas se aumenta gradualmente la temperatura durante el proceso de descarburación. Dado que, al descender el contenido de carbono la temperatura de fusión de la aleación se eleva, la sinterización se verá dificultada.

La idea de partida era efectuar el control de temperatura a partir de los resultados del análisis constante del contenido de carbono (en forma de metano) en la fase gaseosa. Sin embargo, una solución más sencilla consiste en efectuar la descarburación en forma escalonada, determinando el momento justo de efectuar el cambio (aumento) de la temperatura a partir de una serie de experimentos cuyos resultados fueron analizados con la ayuda del modelo establecido por Filippov ${ }^{[16]}$ :

$$
\mathrm{Cf}=\mathrm{Co} \cdot \mathrm{e}^{-\mathrm{kt}}
$$

donde:

Cf - contenido de carbono después de la descarburación, \%;

Co - contenido de carbono antes de la descarburación, \%;

$\mathrm{t}$ - tiempo, h;

$\mathrm{k}$ - constante de régimen que caracteriza la velocidad del proceso.

La evolución del ensayo permite establecer conclusiones precisas acerca del proceso de descarburación. En la figura 2 se presenta un esquema del proceso de descarburación que se sigue a escala industrial. En este, se aprecia que el proceso puede detenerse en un determinado momento con objeto de obtener el contenido deseado de carbono. Los tiempos de retención se pueden calcular empleando los coeficientes $k$ hallados para cada etapa de calentamiento y caracterizan la velocidad de difusión para cada etapa. Estos valores son ${ }^{[17]}$ :

$$
\begin{aligned}
& \mathrm{k}_{\mathrm{I}}=0,099 \\
& \mathrm{k}_{\mathrm{II}}=0,226 \\
& \mathrm{k}_{\text {III }}=0,250
\end{aligned}
$$


Como se aprecia en la figura 4 , el flujo del gas descarburante $f_{a d}$ (en este caso $\mathrm{NH}_{3}$ disociado) también se incrementa a medida que avanza el proceso, ya que esto hace disminuir la presión parcial del metano formado en la interfase gas-sólido, propiciando, también, el desplazamiento del equilibrio de la reacción: $\mathrm{N}_{2}+3 \mathrm{H}_{2} \leftrightarrow 2 \mathrm{NH}_{3}$ hacia la derecha. Los valores de flujo indicados en la figura fueron establecidos a partir de una serie de ensayos llevados a cabo durante la investigación.

El gas descarburante debe ser sometido a un proceso riguroso de desecación, hasta niveles aproximados menores de $30^{\circ} \mathrm{C}$ de punto de rocío, para lo que se hace pasar por una columna de zeolita o algún otro material desecante. De lo contrario, al disociarse a altas temperaturas las moléculas de agua del gas, puede producir la oxidación del polvo, afectando a las etapas posteriores de prensado y sinterización. Importancia especial tiene la oxidación del cromo, debido a que los óxidos de este metal no pueden ser descompuestos con un recocido de reducción posterior.

Otras dos recomendaciones para activar la descarburación son las siguientes:

- La relación entre el volumen del gas contenido en la cámara de descarburación y la masa de polvo debe ser 3:1.

- El polvo debe ser previamente granulado para garantizar el aumento de la superficie de intercambio sólido-gas.

La granulación del polvo se efectúa en un disco peletizador hasta obtener pelets de diámetros entre

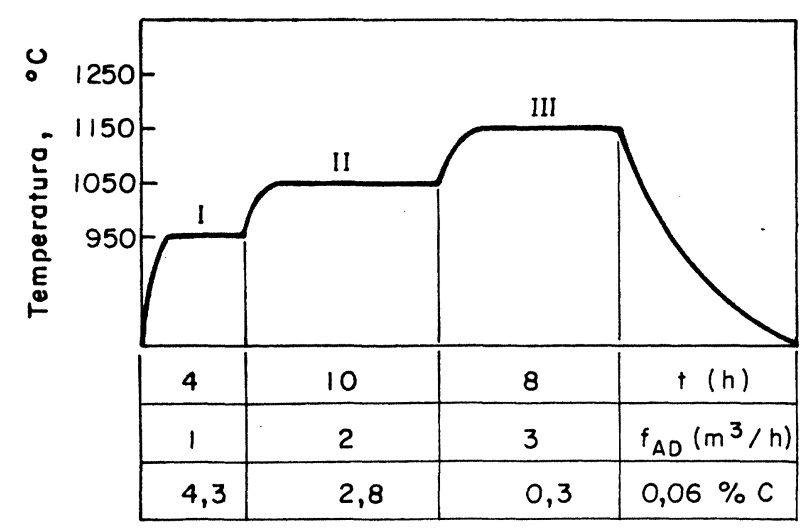

Figura 4. Régimen de descarburación del polvo de acero inoxidable 18-8. La relación de volumen de gas / masa de polvo debe ser 3:1.

Figure 4. Decarburization rate of 18-8 stainless steel powder. The gas volume/powder mass ratio must be 3:1.
5-15 mm. Como aglomerante se emplea alcohol polivinílico diluido aproximadamente al $15 \%$, que se elimina durante la descarburación entre 300 y $350^{\circ} \mathrm{C}$.

El proceso de descarburación se aplicó a escala semi industrial en un horno de cámara con contenedores de acero termoresistente. Sin embargo, a escala de producción, este proceso debe ser realizado en hornos continuos convencionales en los que la altura de la cámara de calentamiento alcanza un tamaño de 150-180 mm, o en hornos especiales diseñados específicamente para este proceso, los cuales han de permitir el incremento de la temperatura y del flujo descarburante, así como la regulación del tiempo de retención.

Como resultado de este proceso, el contenido de carbono disminuye de 4,3 a $0,06 \%$. En la figura 5 se observa la estructura final obtenida, en la que se destaca la alta formación de numerosos poros interños de pequeños tamaños.

\subsection{Molienda}

El ensayo de molienda se realizó con una muestra del polvo de la tercera atomización de tamaño superior a $180 \mu \mathrm{m}$ (fracción gruesa no útil). Esta muestra se sometió a una molienda en un mortero de hierro durante $2 \mathrm{~h}$, tras lo cual se obtuvo polvo de las características que se presentan en la tabla III. Los resultados que se presentan en esta tabla son similares a los que se exponen en la tabla II para la tercera atomización. Los indicadores del

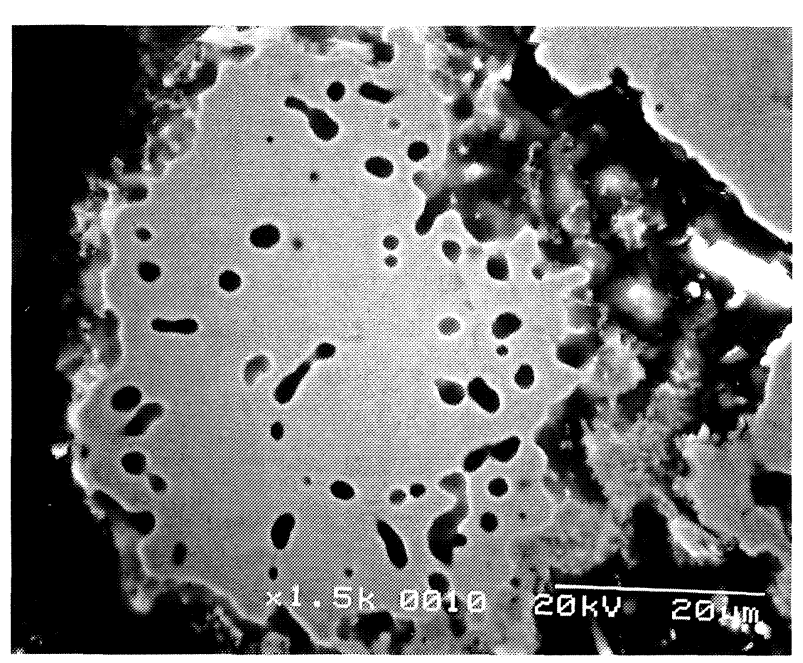

Figura 5. Estructura del polvo descarburizado hasta $0,06 \%$ $\mathrm{C}$, en la que se muestra la presencia de microporosidad interna, $\times 1500$.

Figure 5. Structure of the decarburized powder (until 0,06\% C), showing internal microporosity, $\times 1500$.

Rev. Metal. Madrid 37 (2001) 
Tabla III. Resultados de los ensayos de caracterización de los polvos atomizados tras ser sometidos a un proceso de molienda Table III. Results of the characterization test of atomized powders after milling

\begin{tabular}{cccc}
\hline $\begin{array}{c}\text { Tamaño medio de la fracción, Rendimiento de la fracción } \\
\mu \mathbf{m}\end{array}$ & $\begin{array}{c}\text { Fluidez, } \\
\mathbf{s} / \mathbf{5 0 g}\end{array}$ & $\begin{array}{c}\text { Densidad aparente, } \\
\mathbf{g} / \mathbf{c m}^{\mathbf{3}}\end{array}$ & \multicolumn{1}{c}{ Morfología } \\
\hline 78 & 68 & 3,01 & $\begin{array}{l}\text { Predominio de partículas } \\
\text { irregulares, lisas y redondeadas; } \\
\text { partículas fragmentadas de } \\
\text { tipo irregular }\end{array}$ \\
\hline
\end{tabular}

tamizado (tamaño y rendimiento) permiten concluir que la introducción de esta operación es factible. Se observa que la fluidez y la densidad aparente mejoran ligeramente. En cuanto a la morfología también es muy similar. Esto parece indicar que, en la molienda, se separan partículas aglomeradas durante la atomización.

\section{RESULTADOS Y DISCUSIÓN}

A partir de los resultados obtenidos en la fase experimental, se desarrolló el esquema que se muestra en la figura 6, en la cual se señalan los materiales que se utilizaron para preparar la fusión de la aleación enriquecida de carbono.

Tras la extracción del polvo húmedo de la cámara de atomización, éste se seca en una estufa a $100^{\circ} \mathrm{C}$ durante $1 \mathrm{~h}$. A continuación se tamiza, se extrae la fracción más gruesa para someterla a un proceso de molienda y el producto resultante se mezcla con la fracción más fina. Este proceso se repite varias veces hasta conseguir la homogeneización del polvo. Posteriormente, el polvo homogeneizado se granula $y$, en la etapa siguiente, se descarbura. El polvo descarburado y microporoso es, finalmente, triturado para deshacer los gránulos.

En las tablas IV y V se incluyen las características finales del polvo. Al comparar los resultados hay que tener presente que todas aquellas características relacionadas con la densidad están afectadas por la microporosidad existente en el polvo.

Los valores de fluidez bajos que presenta el polvo (entre 28 y $40 \mathrm{~s} / 50 \mathrm{~g}$ ) respecto a los valores publicados en la literatura ${ }^{[12,13}$ y 18], se explican a partir de la alta rugosidad superficial que adquieren las partículas de polvo durante la descarburación (Fig. 7). La densidad aparente $\left(2,48 \mathrm{~g} / \mathrm{cm}^{3}\right)$ se encuentra dentro del rango de valores $\left(2,3\right.$ y $\left.2,8 \mathrm{~g} / \mathrm{cm}^{3}\right)$ que se encuentra en la literatura ${ }^{[12,13}$ y 18$]$.

En el estudio de compresibilidad realizado con

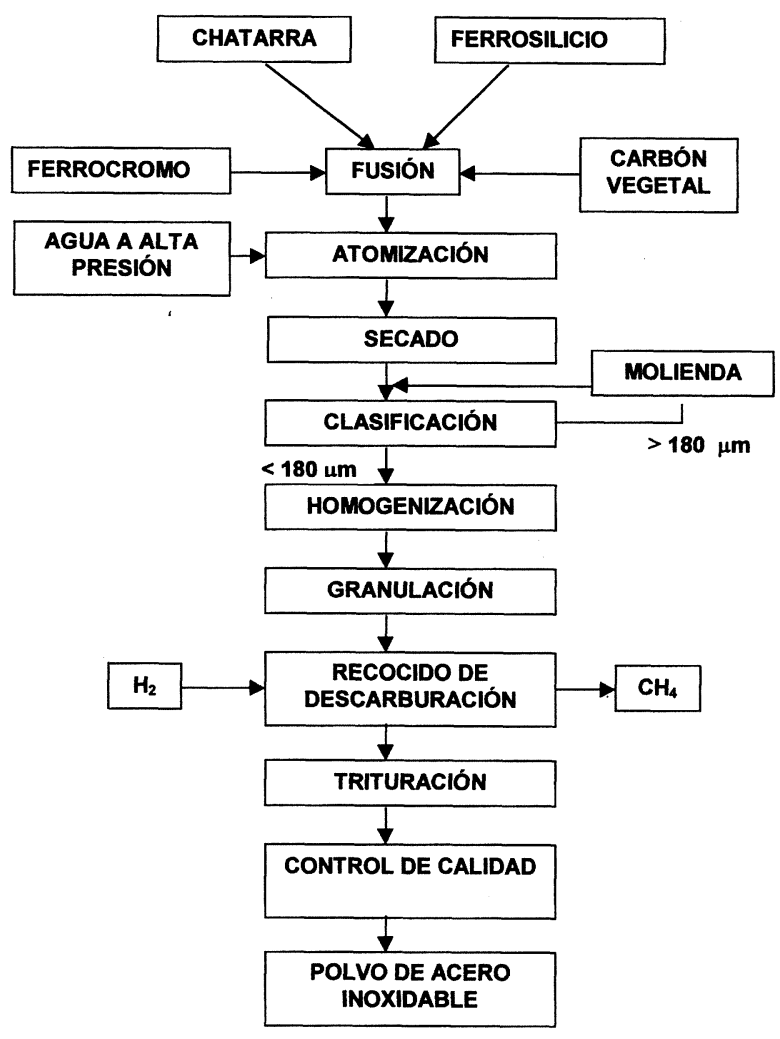

Figura 6. Descripción mediante un diagrama de flujo de las distintas etapas de la tecnología desarrollada para la obtención de polvos de acero inoxidable con estructura microporosa.

Figure 6. Description by a flow diagram of the different steps of the developed technology for the obtention of stainless steel powders with a microporous structure.

el polvo descarburizado se observó que, para presiones de compactación superiores a $450 \mathrm{MPa}$, el polvo es capaz de compactarse sin necesidad de lubricante (estearato de zinc) y, a partir de los $800 \mathrm{MPa}$, se aprecia la aparición de deformación plástica en el compacto. Sin embargo, para el polvo atomizado y sometido a un recocido de restauración 
Tabla IV. Composición química y distribución granulométrica del polvo descarburizado

Table IV. Chemical composition and particle size distribution of decarburized powder

\begin{tabular}{|c|c|c|c|c|c|c|c|c|c|c|c|c|}
\hline \multicolumn{7}{|c|}{ Composición química \% } & \multicolumn{6}{|c|}{ Composición granulométrica } \\
\hline \multirow{2}{*}{ C } & \multirow{2}{*}{ Mn } & \multirow{2}{*}{ Si } & \multirow{2}{*}{$\mathbf{P}$} & \multirow{2}{*}{$\mathbf{S}$} & \multirow{2}{*}{$\mathrm{Cr}$} & \multirow{2}{*}{$\mathbf{N i}$} & \multirow{2}{*}{$\mu \mathbf{m}$} & -180 & -125 & -100 & -75 & \multirow{2}{*}{-37} \\
\hline & & & & & & & & +125 & +100 & +75 & +37 & \\
\hline 0,06 & 0,87 & 1,43 & 0,02 & 0,007 & 18,74 & $\overline{8,0}$ & $\%$ & 24,90 & 18,53 & 27,49 & 20,49 & 8,59 \\
\hline
\end{tabular}

Tabla V. Propiedades físicas de interés tecnológico del polvo descarburizado

Table V. Physical properties with technological interest of decarburized powder

\begin{tabular}{|c|c|c|c|c|c|}
\hline \multirow{2}{*}{$\begin{array}{l}\text { Fluidez } \\
\text { s/50g }\end{array}$} & \multirow{2}{*}{$\begin{array}{c}\text { Densidad } \\
\text { aparente, } \\
\mathbf{g} / \mathrm{cm}^{3}\end{array}$} & \multicolumn{2}{|c|}{$\begin{array}{l}\text { Compresibilidad a } 600 \\
\mathrm{MPa}, \mathrm{g} / \mathrm{cm}^{3}\end{array}$} & \multirow{2}{*}{$\begin{array}{c}\text { Densidad } \\
\text { picnométrica, } \\
\mathrm{g} / \mathrm{cm}^{3}\end{array}$} & \multirow{2}{*}{ Morfología } \\
\hline & & Sin lubricante & Con lubricante & & \\
\hline 56,6 & 2,48 & 5,09 & 5,341 & 7,30 & $\begin{array}{l}\text { Se observa predominio de partículas irregulares, con } \\
\text { superficies rugosas, y presencia de microporos (Fig. 7) }\end{array}$ \\
\hline
\end{tabular}

estructural es necesario aplicar lubricante y presiones superiores a $700 \mathrm{MPa}$ (Tabla II). Por otro lado, al comparar los resultados numéricos (Tabla V) se aprecia que la densidad aparente de $2,48 \mathrm{~g} / \mathrm{cm}^{3}$, está muy por debajo del valor publicado, 6,4-6,8 $\mathrm{g} / \mathrm{cm}^{3}[12$ y 13$]$. Esto se atribuye a la baja densidad que posee el polvo debido a su microporosidad interna.

$\mathrm{Al}$ analizar integralmente la tecnología propuesta en la figura 6 y compararla con la fabricación convencional de polvos de acero inoxidable se aprecian una serie de ventajas:

- Permite emplear hornos de fusión de menor potencia, debido a que el alto contenido de carbono reduce la temperatura de fusión, lo que implica menores consumos energéticos y de refractarios (crisoles).

- Admite el empleo de materias primas de alto carbono disponibles en el mercado nacional y que son más baratas que en el mercado internacional, como por ejemplo, el ferrocromo de alto carbono.

- El uso de la instalación de atomización no limita el rendimiento de la fracción útil, puesto que el polvo se muele fácilmente debido a la fragilidad que adquiere por cuanto se enriquece con carbono durante la fusión.
- Debido al incremento de la microporosidad del polvo durante el recocido, para la compactación se requieren presiones de prensado más bajas, lo que amplía las posibilidades de la conformación y, por tanto, de la capacidad de las prensas.

- Mediante un control adecuado del proceso se pueden obtener aleaciones dentro de un amplio rango de contenido de carbono, lo que permite emplearlo, tanto para obtener aceros inoxidables austeníticos y ferríticos, como martensíticos.

- La fabricación de piezas sinterizadas de acero inoxidable, a partir del polvo previamente descarburado, no se limita a la obtención de filtros sino que también se pueden obtener piezas sinterizadas de formas diversas.

- La descarburación genera subproductos reciclables (hollín, metano) los cuales se pueden emplear en otros procesos pulvimetalúrgicos.

La desventaja del procedimiento es la introducción necesaria del recocido de descarburación. Sin embargo, el costo adicional que supone este paso intermedio, sobre el costo total de producción de la tonelada de polvo de acero inoxidable, se justifica de acuerdo a los precios del polvo en el mercado internacional. En la tabla VI se pueden apreciar 
Tabla VI. Análisis comparativo de los diferentes parámetros de procesado involucrados en la tecnología propuesta y la convencional

Table VI. Comparative analysis of the different processing parameters involved in the conventional and proposed technologies

\begin{tabular}{lcc}
\hline Indicadores & $\begin{array}{r}\text { Procedimiento } \\
\text { convencional }\end{array}$ & $\begin{array}{c}\text { Procedimiento } \\
\text { propuesto }\end{array}$ \\
\hline $\begin{array}{l}\text { Temperatura de } \\
\text { atomización, }{ }^{\circ} \mathrm{C}\end{array}$ & $1.650-1.750$ & $1.550-1.650$ \\
$\begin{array}{l}\text { Presión del agua, } \mathrm{MPa} \\
\text { Rendimiento de polvo }\end{array}$ & 14 & $8-10$ \\
$\begin{array}{l}\text { útil,\% masa } \\
\text { Microporosidad interna, } \\
\% \text { volumen }\end{array}$ & 70 & Hasta 100 \\
$\begin{array}{l}\text { Presión de compactación, } \\
\text { MPa }\end{array}$ & $550-830$ & $6-10$ \\
\begin{tabular}{l} 
Ganancia económica, \% \\
\hline
\end{tabular} & 100 & 120 \\
\hline
\end{tabular}

las diferencias entre la tecnología convencional y la propuesta.

\section{CONCLUSIONES}

- El proceso propuesto permite la obtención de polvo de acero inoxidable, del tipo 304, con características químicas, físicas y tecnológicas comparables a los que se encuentran en la literatura y en correspondencia con su estructura microporosa.

- La introducción del carbono en el metal durante la fusión hasta niveles del orden de 4,3\%, garantiza:

- la formación posterior de la estructura microporosa mediante un recocido bajo atmósfera descarburante

- la fragilidad del polvo atomizado permite una reducción significativa de los costos debido a que se puede conseguir un rendimiento del material del $100 \%$ cuando se muele la fracción no útil

- se reduce notablemente la temperatura de fusión, lo que disminuye el consumo de energía y material refractario de los crisoles

- se prescinde de materias primas de bajo carbono, lo que permite el empleo de los recursos materiales de menor costo.
- La tecnología desarrollada para la obtención de polvos microporosos de acero inoxidable se optimiza con la introducción de:

- molienda de la fracción gruesa

- granulación del polvo

- ajuste del régimen escalonado de recocido de descarburación para conseguir una reducción significativa de los costos.

- La tecnología desarrollada presenta una serie de ventajas que la hacen competitiva entre las tecnologías convencionales. Estas ventajas se resumen en la posibilidad de uso de materias primas nacionales y la reducción de las exigencias tecnológicas para la obtención y prensado de los polvos de acero inoxidable.

\section{Agradecimientos}

Los autores desean destacar la valiosa colaboración de investigadores de diversos países que nos han ofrecido desinteresadamente sus publicaciones. Un agradecimiento especial al Dr. Juan A. Bas Carbonell, al Dr. César Molins y su empresa AMES, S.A. por su inestimable ayuda en información y con el estudio microscópico. Un reconocimiento a los investigadores y técnicos del CIME y CENIM, así como a los estudiantes del ISPETP y de otras instituciones por su participación en los trabajos experimentales.

\section{REFERENCIAS}

[1] A.F. SHORniak y V.E. Olikier, Poroshk. Metall. 29 (1977).

[2] A.V. Rabinovich y Y.B. ZaslovskiI, Metalloved. Term. Obrab. Met. 3 (1985).

[3] Patente Reino Unido 762.267, Federal - Mogul Corp., Porous sintered articles of austenitic stainless steel, 1956.

[4] Patente Alemania 938.428, Method for production of formed parts from sintered alloy steel, Gebr Böhler \& Co. A-G, 1956.

[5] K. Sadakimi, Patente Japón 02057604 A, Production of injection molding powder and metallic sintered body, 1990.

[6] K. SADAKIMI, Patente Japón 02057607 A, Injection molding powder and production of metallic sintered body, 1990.

[7] H. TAKEO, Patente Japón 03079703 A, Manufacture of stainless steel sintered product and stainless steel powder, 1991.

[8] R.M. LARSEN y K.A. Thorsen, Removal of oxygen and carbon during sintering of austenitic Stainless steel, P/M World Congress, Japan Society of Powder and Powder Metallurgy, Kyoto, Japón, 1993, pp. 377-380. 
[9] O. KuROAKI, Patente Japón 05271713 A, Method for sintering alloy containing easily oxidizable element, 1993.

[10] J. SAUNDERS, WO 96/28580, Stainless steel powders and articles produced therefrom by PM, 1995.

[11] R.L. Probst y G.J. Le Brasse, Patente EE.UU. 2.826.805, to Federal - Mogul Corp., Sintered stainless steel alloys, 1958.

[12] ASM. Production of stainless steel low - alloy steel and tool steel, Metals Handbook, $9^{\text {th }}$ Ed., vol. 7, 1988, pp. 100-104.

[13] R.W. StEVEnSON, P/M stainless steel, Metals Handbook, $9^{\text {th }}$ Ed., vol. 7, 1988, pp. 728-732.
[14] N. Krivij, W. Suwardjo, L. García, A. Cores y A. Formoso, Rev. Metal. Madrid 33 (1997) 229-238.

[15] L. Nyborg, Surf. Interface Anal. 19 (1992) 607-614.

[16] F.I. FiLIPPOV, The theory of metallurgical processes, Ed. MIR, Moscú, Rusia, 1975, p. 296.

[17] M. MARTínEZ, y W. SuWARDjo, II Encuentro de Ingeniería de Materiales, ISPJAE, La Habana, Cuba, 1998, pp. 155 160.

[18] Fukuda Metal Foil and Co. Ltd., Met. Powders 12 (1994). 